\title{
Logics of Informational Interactions
}

\author{
Alexandru Baltag • Sonja Smets
}

Received: 26 June 2014 / Accepted: 1 July 2014 / Published online: 26 April 2015

(C) The Author(s) 2015. This article is published with open access at Springerlink.com

\begin{abstract}
The pre-eminence of logical dynamics, over a static and purely propositional view of Logic, lies at the core of a new understanding of both formal epistemology and the logical foundations of quantum mechanics. Both areas appear at first sight to be based on purely static propositional formalisms, but in our view their fundamental operators are essentially dynamic in nature. Quantum logic can be best understood as the logic of physically-constrained informational interactions (in the form of measurements and entanglement) between subsystems of a global physical system. Similarly, (multi-agent) epistemic logic is the logic of sociallyconstrained informational interactions (in the form of direct observations, learning, various forms of communication and testimony) between "subsystems" of a social system. Dynamic Epistemic Logic (DEL) provides us with a unifying setting in which these informational interactions, coming from seemingly very different areas of research, can be fully compared and analyzed. The DEL formalism comes with a powerful set of tools that allows us to make the underlying dynamic/interactive mechanisms fully transparent.
\end{abstract}

Keywords Dynamic epistemic logic · Quantum logic · Logical dynamics

\section{Introduction}

Our contribution to this anniversary issue of the Journal of Philosophical Logic is meant to give a glimpse into the work done in the last years on logics of informational

\footnotetext{
A. Baltag $(\bowtie) \cdot$ S. Smets

ILLC, University of Amsterdam, Amsterdam, The Netherlands

e-mail: thealexandrubaltag@gmail.com

S. Smets

e-mail: S.J.L.Smets@uva.nl
} 
interactions, with a particular stress on two research streams: Dynamic Epistemic Logic(s) and Quantum Dynamic Logic. These lines of research are just some of the most recent and on-going developments within the wider (and older) trend known as the "dynamic turn in logic", trend pursued (mainly, but not exclusively) by the Amsterdam school of logic, trend represented (among others) by Johan van Benthem, Frank Veltman and Martin Stokhof. In this paper, we use the term "informational interactions" to refer to a range of processes belonging to different fields of study encompassing dialogues, observations, physical measurements, acts of learning as well as communication. In fact, the systematic study of informational interactions ties in with older developments on action-based reasoning in the areas of theoretical Computer Science, Philosophy as well as Quantum Logic and Computation. In Computer Science, action-based reasoning has seen the development of systems such as Hoare logic, Dynamic Logic, Labeled Transition Systems, Petri Nets, Automata Theory etc. In Formal Epistemology, various models for belief revision and knowledge updates have been introduced to model situations in which the knowledge or belief states of an agent change in the face of new incoming information. In the Logical Foundations of Quantum Physics, the work on operational quantum logic treats experimental procedures as the basic building blocks used to describe the behavior of quantum systems. These areas might seem far apart at first sight, as indeed that is how they have been treated for a long time, but a closer look at the involved "informational interactions" reveals that they share a common dynamic/interactive nature.

In the next section we argue that Quantum logic can be viewed as the logic of physically-constrained informational interactions and similarly how multi-agent epistemic logic can be viewed as the logic of socially-constrained informational interactions. Instead of working with purely static propositional formalisms, we show that the fundamental operators at play, are really dynamic in nature: quantum implication is best understood as a form of dynamic "weakest precondition" modality (as in Propositional Dynamic Logic [30]), that pre-encodes the result of possible measurements; quantum negation is a special case of this, pre-encoding the impossibility of obtaining certain measurement results; while "knowledge" is best understood as the result and the object of "epistemic activity", which is a special kind of belief management (namely one that is truth-directed). Dynamic Epistemic Logic, originally designed to model and reason about socially-constrained informational interactions, offers a powerful set of tools that can well be applied to physicallyconstrained informational interactions as well. These views allow us to draw connections between different areas, making explicit their underlying dynamic/interactive nature.

In Section 2 we introduce the background and main ideas of Dynamic Quantum Logic. In Section 3 we introduce the key features of Dynamic Epistemic Logic and explain the link to belief revision theory. In Section 4 we draw connections between the two different frameworks. We conclude with a few indicators of promising new developments that we see in this area of research. 


\section{Dynamic Quantum Logic}

The dynamic perspective on quantum logic connects well with the earlier investigations on operational quantum logic, i.e. the "manual" approach (or so-called test spaces) of Foulis and Randal [27, 37] and the work of the "Geneva School" led by Jauch and Piron [31-34]. This view equips quantum logic with an operational dimension, linking every physical property or proposition about a physical system to the experimental procedures that can be performed on those systems. Inspired by the work of C. Piron, [33, 34], the operational view has lead to new axiomatic systems that are now studied in the context of Dynamic Quantum Logic $[7-9,12-14]$. In contrast to the traditional work on quantum logic (following [24, 39]), the dynamic logical approach has several advantages. Its main value lies in the fact that all the qualitative non-classical properties of quantum systems become explainable in terms of the non-classical flow of quantum information. The essence of quantum non-classicallity lies in the non-classical nature of quantum actions, and not in the failure of the classical laws of static propositional logic. In a long-standing debate (sparkled by a discussion in [36]) many held the opposite view, which according to us is due to a misunderstanding about the logical laws at play in the quantum world. In our account, quantum mechanics does not require any modification of the classical laws of propositional logic. The classical laws of propositional logic, modelling the static propositions, are fully compatible with dynamic quantum logic. Another virtue of this approach is that it explicitly bridges the gap between traditional quantum logic and quantum computation, yielding a basic logical tool that can be further adjusted to formally verify the correctness of quantum protocols and algorithms.

As a formal framework, Dynamic Quantum Logic treats quantum actions differently from the propositions that express static properties about a physical system. The central force of the framework builds on a distinction between two types of quantum actions: the first are called "tests" of physical properties and correspond to successful yes-no measurements while the second are called "quantum gates" and correspond to the logical gates via which we can let a system evolve. These quantum "tests" are meant to represent successful measurements of some yes/no property (call it $\varphi$ ). As such, the quantum test of $\varphi$ expresses that if property $\varphi$ is tested, the answer is positive and consequently the state of the (observed) system will collapse to a state exhibiting property $\varphi$. In this way we view quantum tests actions as forms of information update. In contrast to tests, the quantum gates represent reversible evolutions of the physical system.

In Section 4, we compare the quantum test to other dynamic-informational operators in logic and highlight their common features and differences. But before we get to this comparison, it is crucial to see that quantum tests exhibit a blending of both ontic and epistemic features. The ontic features of quantum tests tie in with the appearance of what is known as a "physical observer effect" when we measure quantum systems. Quantum test actions can (and typically also do) change the ontic state 
of the "observed" system. So any epistemic action by an observer, used to extract (or learn) information from a quantum system, may have ontic side-effects. In [10], we formulated this point as a slogan: in a quantum universe "there is no information change without changing the world". The epistemic features of quantum tests refer to the increased uncertainty we experience due to measuring incompatible physical properties (such as for example, position and momentum). After consecutively testing two incompatible properties (first test $\varphi$ and then $\psi$ ), the acquired information about $\varphi$ gets overwritten by testing for $\psi$. This feature explains the non-monotonic dynamics of quantum information change. Note also that in the quantum world, the ontic effects of quantum tests can be non-local. This refers to the fact that quantum tests are local actions, used to gather local information about one part of a system. Such local actions can induce "non-local" ontic effects on another, remote part of the system. The latter feature of quantum information flow is crucial for our understanding and modeling of the notion of quantum entanglement. Quantum entanglement is traditionally defined in Quantum Theory in a quantitative manner, via linear combinations and tensor products. However, in our dynamic approach we capture entanglement in a different way, by using pure qualitative tools. In [8-10, 12], we added (qualitative) spatial features to dynamic quantum logic, allowing us to talk about the local properties of given subsystems of a quantum system. We will illustrate the formal details of this in the next section.

\subsection{Formalism of Dynamic Quantum Logic}

Dynamic Quantum Logic adopts the language of Propositional Dynamic Logic $(P D L)$, consisting of a level of formulas $\varphi$ and programme constructs $\pi$, defined by mutual recursion:

$$
\begin{array}{lll|l|l|l|l}
\varphi & ::= & \perp & p & \neg \varphi & \varphi \wedge \psi & {[\pi] \varphi} \\
\pi & ::= & a & \mid & \varphi ? & \pi \cup \pi & \pi ; \pi
\end{array}
$$

The language is build up from atomic sentences $p$ belonging to a given set of atomic formulae and basic actions $a$ belonging to a given set of actions. We work with the standard operators for classical negation $\neg$, conjunction $\wedge$, tests $\varphi$ ?, arbitrary choice of actions $U$, action composition; and the dynamic construct $[\pi] \varphi$. While the language is known from the work on $P D L$, its semantics is different. In this formalism a non-classical semantics is given in terms of non-classical relational models, called Quantum Transition Systems (QTS). In [7], we first illustrate how Hilbert spaces can be structured as QTS's. The work in [7] was the start of a new semantic approach, which was later further developed in a series of papers [8, 9, 12-14]. In the next paragraph we introduce the setting of Quantum Transition Systems (QTS) as part of our quantum semantics for the language of $P D L$.

A QTS consist of a set $\Sigma$ of states, a family of labeled transition relations $\{\stackrel{P ?}{\rightarrow}\} \subseteq \Sigma \times \Sigma$ where the labels represent test actions that come from a given set of testable properties $\mathcal{L} \subseteq P(\Sigma)$, and a family of labeled transition relations $\{\stackrel{U}{\rightarrow}\} \subseteq \Sigma \times \Sigma$ where the labels $U$ represent quantum gates. These two types of binary relations correspond to the two types of basic actions: "quantum tests" 
denoted by $P$ ? and "quantum gates" denoted by $U$. In the Hilbert space formalism, the "states" correspond to rays (or one-dimensional subspaces) of a given Hilbert space $H$. A quantum test $\varphi$ ? corresponds to a projector onto (the subspace generated by) property $\varphi$ in $H$. Quantum gates $U$, represent reversible evolutions, corresponding to unitary transformations in $H$.

The negation-free part of the above language of $P D L$ can be interpreted in the semantics given by QTS. This can be done by interpreting tests $\varphi$ ? as quantum tests and basic actions $a$ as quantum gates. The program expressions $\pi$ are interpreted as quantum programs which can be compound constructions building on the basic ingredients of quantum tests and quantum gates. The other operators behave as expected, in particular $\wedge$ is the classical conjunction and the dynamic construct $[\pi] \varphi$ is used to express the weakest precondition: it captures that if program $\pi$ would be performed on the current state of the system then the output state will satisfy $\varphi$. The non-classical features of quantum systems arise in this setting only due to the nonclassical behavior and interpretation of the quantum actions (and their compounds) $\pi$. This logical system obeys the principle of bivalence. Traditional quantum logic (or "orthomodular logic" ) can then be re-interpreted inside this logic, first by defining the operation of orthocomplement in terms of the given basic constructs. Note that in our dynamic approach the orthocomplement $\sim \varphi$ of a property $\varphi$ can be viewed as the impossibility of a successful quantum test, hence we can define $\sim \varphi:=[\varphi$ ?] $\perp$. The quantum join (or quantum disjunction) is then definable via de Morgan dual of the classical conjunction under orthocomplement, i.e. $\varphi \sqcup \psi:=\sim(\sim \varphi \wedge \sim \psi)$. The "quantum implication" (or so-called Sasaki hook) is elegantly given by the weakest precondition of a "quantum test": $\varphi \stackrel{S}{\rightarrow} \psi:=[\varphi$ ? $\psi \psi$.

The interpretation for the operators in the full language of $P D L$ (with classical negation), demands a slightly different semantic setting. In this case we work with a generalized QTS $[10,11]$, i.e. a QTS in which the only change is that tests $\{\stackrel{P ?}{\rightarrow}\}_{P \subseteq \Sigma}$ are labeled by arbitrary properties (sets of states) instead of testable properties in $\mathcal{L}$. Note that in Hilbert spaces these arbitrary properties (or propositions) do not necessarily correspond to a closed linear subspace, while the testable properties do. However the actions of the form $\stackrel{P \text { ? }}{\rightarrow}$ in a generalized QTS still represent "quantum tests", which are now defined as the test of the strongest testable property that is entailed by $P$ (because indeed $P$ itself might not be testable). In Hilbert spaces this will correspond to the closed linear subspace that is generated by the set $P$. The interpretation of the language constructs of $P D L$ in a generalized QTS is straightforward, using the classical set-theoretic complement to give an interpretation to the classical negation. Note that the full setting of this logic (which includes the classical negation) was called the logic of quantum actions (LQA) in [10, 11]. It is important to note that $L Q A$ has more expressive power than standard quantum logic due to the presence of the classical negation $\neg$ in its language. In $[10,11]$ we point to the construction of formulas in $L Q A$, expressing quantum properties of a physical system, that have no counterpart in traditional quantum logic.

In $[9,12]$, we added (qualitative) spatial features to dynamic quantum logic, allowing us to express local properties of given subsystems of a quantum system. Starting from the above (generalized) QTS semantics, we now extend the setting in 
order to express that the actions can be of various types, depending on their location. As an example we consider a given compound system $\mathcal{S}$, composed of two subsystems called $\mathcal{S}_{1}$ and $\mathcal{S}_{2}$. The (generalized) QTS for system $\mathcal{S}$ needs to include, besides the "global" actions $U$ and test $P$ ? (on the whole system), also $i$-local quantum gates $U_{i}$ and $i$-local quantum tests $P_{i}$ ? (for $i=1,2$ ) that can be performed only on one of the subsystems $\mathcal{S}_{i}$. There are several ways in which this idea can be further implemented, following the ideas in [12] we proceed by equipping our (generalized) QTS with a binary equivalence relation $\simeq_{i}$ over (global states) in $\Sigma$. Two possible global states $s, s^{\prime} \in \Sigma$ of system $\mathcal{S}$ are "indistinguishable" from the point of view of subsystem $\mathcal{S}_{i}$ iff $s^{\prime}$ can be obtained from $s$ by performing a unitary action $U$ that affects only the environment but leaves $\mathcal{S}_{i}$ intact. For two global states of our given compound system $\mathcal{S}$, we set $s \simeq_{1} s^{\prime}$ iff there exists a 2-local unitary action $U_{2}$ such that $s^{\prime}=U_{2}(s)$. The local state $s_{i}$ of $\mathcal{S}_{i}$ (when the global state is $s$ ), corresponds to the $\simeq_{i^{-}}$ equivalence class given by $s_{i}:=\left\{s^{\prime}: s \simeq_{i} s^{\prime}\right\}$. We can view an $i$-local state as a set of those global states that are informationaly consistent with the $i$ th-subsystem. Taking the concept of an $s_{i}$-local state as our basic ingredient, one can define a notion of an $i$-local property $\varphi_{i}$ in $s$. So $s$ satisfies $\varphi_{i}$ iff the state $s$ is separated in two parts, an $i$-part and $\neg i$-part, where the property $\varphi$ is true only in the states that are $i$-equivalent to $s$. An $i$-local test is then taken to be the test of an $i$-local property. We briefly note that there are alternative ways to define the $i$-equivalence relation $s \simeq_{i} s^{\prime}$. For example, instead of working with unitary operators one can express a correlation between the outcomes of the quantum measurements on subsystems. In that case we have to assume that for every $i$-local measurement, the probability of obtaining any given result is the same in state $s$ as in state $s^{\prime}$.

As standard in modal logic, we introduce a Kripke modality $K_{i}$ as the operator for the added equivalence relation $\simeq_{i}$. For every property $\varphi \subseteq \Sigma$ and every component $i \in N$ (where $N$ are the labels for subsystems), we define:

$$
K_{i} \varphi:=\left\{s: t \in \varphi \text { for all } t \simeq_{i} s\right\}=\left\{s: s_{i} \subseteq \varphi\right\} .
$$

We read $K_{i} \varphi$ as a "spatial" operator capturing that the information that (the global system satisfies) $\varphi$ is potentially available at location $i$.

Entanglement can now be expressed in our logic via the non-local features we have introduced. We focus on expressing the existence of an "informational correlation" (without communication) between the subsystems to characterize the notion of entanglement. First note that a global state $s$ is separable (or, more precisely, subsystem $\mathcal{S}_{1}$ is separable in the state $s$ ) iff there exists a state $s^{\prime}$ such that $s \simeq_{2} s^{\prime} \simeq_{1} c$ (where $c$ is the special constant for a known separable global state). Otherwise, the state $s$ is said to be entangled. Using our $K_{i}$ modality, we can give an"epistemic" characterization of entanglement as follows:

$s$ is entangled iff it satisfies the sentence $K_{2} K_{1} \neg c$. In other words, two physical systems are entangled if and only if they potentially carry (non-trivial) information about each other (prior to any communication).

As such this setting allows for the study multi-partite quantum information flow and can be used to provide a formal logical analysis of various protocols in quantum computing $[9,13,14]$. 


\section{Dynamic Epistemic Logic}

Dynamic Epistemic Logic (DEL) was originally designed to deal with complex multi-agent learning and communication scenarios in which individual or groups of agents update their knowledge. Typical scenarios include the muddy children puzzle (see e.g. [26]) in which a group of agents gain knowledge about the real state of affairs after a series of truthful public announcements. In cases where all such "informational interactions" between agents are of the form of truthful public announcements, the tools of Public Announcement Logic (PAL) can be applied. PAL is one of the logical systems belonging to the DEL framework which was first formalized (via the use of Reduction Laws) by Plaza [35] and independently by Gerbrandy and Groeneveld [29]. Note that the problem of completely axiomatizing PAL in the presence of a common knowledge operator was first solved by Baltag, Moss and Solecki [5]. In PAL, we equip a multi-agent Kripke model with additional modal operators to model actions. These modal operators for actions are labeled both by propositions and the so-called "public announcement" operator "!". The action ! $\varphi$, when successful, has the effect of deleting the non- $\varphi$ worlds in a given model, and represents the event by which all agents jointly learn that $\varphi$ was true before the public announcement. As a consequence of such changes in a given model, the very property that was announced, while true before the announcement may become false afterwards. This happens for instance in the case when the sentence that is announced to agent $a$ is a Moore sentence of the form $p \wedge \neg K_{a} p$ (stating that $p$ holds but $a$ does not know $p$ ). This Moore sentence while true before the announcement becomes false afterwards (as the second conjunct is then no longer true). For this reason we stress that a successful learning act under a truthful pulic announcement can only guarantee that agents learn that something was true before the announcement but not necessarily afterwards. However, the state of the observed system, capturing the ontic state of affairs or so-called "facts", is not affected by these public announcements. In DEL this is captured by the idea that atomic formulas, are left invariant under the announcements. ${ }^{1}$

Not all "informational interactions" are announcements and not all announcements are public. A logic for "secret (fully private) announcements" was first proposed by Gerbrandy [28] and a logic for "private, but legal, announcements" (or what has been called "fair-game announcements") was developed by H. van Ditmarsch [25]. Further, PAL has been extended with general announcement operators for "epistemic action types" in the work of Baltag and Moss [4]. These action types capture besides public announcements also various other actions including private secure communication. Such epistemic action types were first introduced to model various forms of "knowledge updates", i.e. situations in which the agents face new information that is consistent with their prior information state. Later developments go beyond this setting and include ideas from belief revision theory. In the context of beliefs, agents

\footnotetext{
${ }^{1}$ DEL can also deal with so-called (ontic) fact-changing operations, see [2, 23]. Note that such fact changing operations in the epistemic/doxastic setting of DEL require additional features and are not modelled as an effect of a pure epistemic action (such as a public or private announcement).
} 
update or revise their prior (belief) state with new information that may actually contradict their prior beliefs. In this context, traditional DEL and the standard AGM framework on belief revision [1] have been merged into a new study that extends both approaches [15-18, 20-22].

\subsection{Formalism of Dynamic Epistemic Logic}

The toolbox of standard DEL, uses Multi-agent Kripke models (see e.g. [6]). Starting from a given set $\Phi$ of basic (non-epistemic) facts and a finite set $\mathcal{A}$ of agents, a $\Phi$ Kripke model is a triple $\mathbf{S}=(S, \stackrel{\mathcal{A}}{\rightarrow},\|\|$.$) consisting of a set S$ of states (or possible worlds); a family of binary accessibility relations $\stackrel{a}{\rightarrow} \subseteq S \times S$, one for each agent $a \in \mathcal{A}$, and a valuation $\|\cdot\|: \Phi \rightarrow \mathcal{P}(S)$ assigning to each $p \in \Phi$ a set $\|p\|_{\mathbf{S}}$ of states. The valuation is also called a truth map and is meant to express the factual content of a given world, while the arrows $\stackrel{\mathcal{A}}{\longrightarrow}$ express the agents' epistemic uncertainty between various worlds. As standard, we write $s \models_{\mathbf{S}} \varphi$ for the satisfaction relation: $\varphi$ is true at world $s$ in model $\mathbf{S}$.

For every sentence $\varphi$, we can define a sentence $\square_{a} \varphi$ by (universally) quantifying over the accessible worlds: $s \models_{\mathbf{S}} \square_{a} \varphi$ iff $t \models_{\mathbf{S}} \varphi$ for all $t$ such that $\stackrel{a}{\rightarrow} t$. Here, $\square_{a} \varphi$ may be interpreted as knowledge (in which case we use the notation $\mathcal{K}_{a} \varphi$ instead) or belief (in which case we use $B_{a} \varphi$ instead), depending on the context. The existential dual operator $\nabla_{a} \varphi:=\neg \square_{a} \neg \varphi$ denotes a sense of "epistemic/doxastic possibility".

A doxastic model (or $K D 45$-model) is a $\Phi$-Kripke model satisfying the following properties: seriality, i.e. for every $s$ there exists some successor $t$ such that $s \stackrel{a}{\rightarrow} t$;

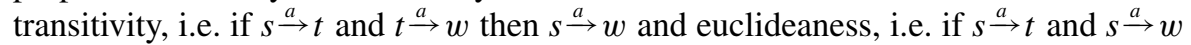
then $t \stackrel{a}{\rightarrow} w$. In a doxastic model, $\square_{a}$ is interpreted as belief, and denoted by $B_{a}$.

An epistemic model (or $S 5$-model) is a Kripke model in which all the accessibility relations are equivalence relations, i.e. reflexive, transitive and symmetric. In an epistemic model, $\square_{a}$ is interpreted as knowledge $\mathcal{K}_{a}$. While $S 5$ yields an elegant logic for reasoning about distributed systems in AI, epistemologists have debated its properties in the context of modelling knowledge. Various alternative notions of knowledge have been suggested and can be formalized in DEL [3]. An important weaker notion of knowledge $(S 4)$ can be modeled via a reflexive and transitive accessibility relation but gives up symmetry or Euclideaness (this notion is then truthful and positively introspective but not necessarily negatively introspective).

DEL uses Kripke models (called event models) epistemic events, including the agents' knowledge/beliefs about the current event, in a similar manner to the way Kripke models are traditionally used to represent the agents' knowledge/beliefs about the current state of the world. An event model (or "action model") $\Sigma=(E, \stackrel{\mathcal{A}}{\rightarrow}$, pre $)$ consists of a set $E$ of "epistemic events" or actions, a precondition map pre associating a sentence pr $_{e}$ to each event $e$, and binary accessibility relations $\stackrel{\mathcal{A}}{\rightarrow}$ for every agent. The events $e \in E$ are assumed to be purely epistemic actions (e.g. observation, testimony, various other forms of communication or learning); pre $e_{e}$ is the precondition of the action $e$, i.e. a sentence that is true in a possible world iff action $e$ can be performed in that world. The accessibility relations express the the current event's 
"appearance" to each participant: the agents' knowledge (or beliefs) about the current action taking place.

A final key ingredient of DEL is the so-called Product Update mechanism, which allows us to compute the effect of events on any initial epistemic/doxastic state models. Given an input state model $\mathbf{S}=(S, \stackrel{\mathcal{A}}{\longrightarrow},\|\|$.$) and an event model$ $\Sigma=(E, \stackrel{\mathcal{A}}{\rightarrow}$, pre $)$, we define their update product $\mathbf{S} \otimes \Sigma=(S \otimes E, \stackrel{\mathcal{A}}{\rightarrow},\|\|$. to be a new (updated) model, containing the set of states $S \otimes E$ given by

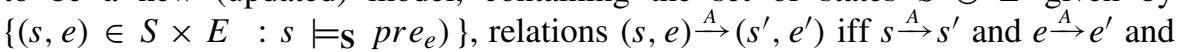
valuation $\|p\|_{\mathbf{S} \otimes \Sigma}=\left\{(s, e) \in S \otimes E: s \in\|p\|_{\mathbf{S}}\right\}$. So two output-states are epistemically indistinguishable iff they are the result of indistinguishable actions performed on indistinguishable input-states. This construction is based on two intuitive principles about rational knowledge change: 1) the "No Miracles" Principle, saying that new knowledge can only be gained from epistemic (features of) actions; and 2) the "Perfect Recall" Principle, saying that knowledge once gained is never lost. Finally, the fact that the new valuation in the updated model is the same as in the input-model reflects the "classical" idealizations assumed in standard DEL: in such a classical setting (unlike a quantum setting), actions such as announcements, testimony and learning can be assumed to be "purely epistemic", i.e. to have no direct ontic effects.

Note that the update product works well when dealing with "hard information" (absolutely certain and infallibly true knowledge), or even with (possibly false) beliefs, as long as these beliefs are never contradicted by new incoming information. However, if the incoming information goes against any prior beliefs, the update product gives unintuitive results: in this case, the agent acquires inconsistent beliefs! In order to avoid this undesirable outcome, several "softer", more belief-revisionfriendly versions of DEL have been developed in recent years, by borrowing ideas from Belief Revision Theory.

The standard theory of AGM belief revision adopts a number of postulates governing "rational" belief revision. In the AGM setting, the initial doxastic state is given by a deductively closed set of sentences $T$, encoding the prior belief set (or theory) of an agent. The action $* \varphi$ of "revision with $\varphi$ " changes the prior theory $T$ into a revised set $T * \varphi$. The AGM postulates impose conditions on the revision operator $*$, essentially incorporating the idea that revision is always successful $(\varphi \in T * \varphi)$ and it changes the agent's doxastic state in a minimal way (preserving "as much as possible" of her prior beliefs). The DEL-style formalism provides a modal-logic incarnation of the AGM framework, in terms of conditional reasoning or contingency plans for belief revision [18]. In a possible world $s$, saying that an agent $a$ believes $\varphi$ conditionally on $\psi$ (written as $B^{\varphi} \psi$ ) means that: agent $a$ 's revision plan is such that, if she were to learn $\psi$, then she would come to believe that $\varphi$ was the case (in the initial state, before the learning). In essence this expresses a conditional or hypothetical, "static" form of revision. In contrast, dynamic belief revision captures the revised beliefs about what is true in the state after the learning action. The belief-revision friendly setting of DEL $[17,18,21,22]$ can handle both types of changes (i.e. static and dynamic revision), where dynamic revision is modeled via dynamic modalities for specific epistemic events (corresponding to specific "model transformers"). 


\section{The Logic of Informational Interactions}

Our conclusion is that, both in the logical foundations of quantum mechanics and in the logical approach to formal epistemology, the key notions and operators have a fundamental dynamic nature. From this perspective we can compare the different types of logical dynamics characterizing these different areas, and analyze their points of agreement and difference.

In line with our extended analysis in [10], we observe that quantum test operators $\varphi$ ? share some common features with public announcements $\varphi$ ! and AGM belief revision $* \varphi$. All these operators represent informational actions, by which either a classical or quantum information state gets updated with new information $\varphi$. First, note that when a testable property is actualized by a successful quantum test $\varphi$ ?, the observed property becomes true after the test: the observed system changes in such a way that it come to satisfy the observed property $\varphi$ (although it may have failed to satisfy this property before the test). We see a similar behaviour in the revision operator $* \varphi$ : here, $\varphi$ becomes accepted by the agent after a successful revision with $\varphi$ (although the agent may have not accepted $\varphi$ before the revision). However, this is not the case for truthful public announcements. We refer here for instance to the Moore sentence $p \wedge \neg K p$, which even if initially true becomes false after it is announced to the (implicit) agent. Such Moore sentences are typical examples of sentences that cannot be successfully learned: they are falsified by the very act of being learnt.

Second, observe that a quantum test can only be successful if the tested property was "potentially true" before the test (i.e. the original state was not orthogonal to $\varphi$ ). This is different from the case of successful (truthful) announcements, whose precondition is that $\varphi$ was actually true before the announcement (in the original state). Third, another similarity between belief revision and quantum observations is that quantum tests affect the original state in the minimal possible way that is compatible with satisfying the observed property. Indeed, in quantum mechanics the state of the observed system after a measurement $\varphi$ ? is the minimal modification of the original state that has the tested property $\varphi$. Similarly, authors in the Belief Revision literature justify the AGM postulates and other, stronger conditions by assuming a "conservatism" principle: the revision with $\varphi$ should require only the minimal doxastic change that is compatible with accepting the new information $\varphi$.

Fourth, like all other forms of information update, quantum tests have epistemic effects. After a successful quantum test $\varphi$ ? is performed, new information becomes accessible to the observer: we have not only $[? \varphi] \varphi$, but also $[? \varphi] K \varphi$. Fifth, quantum tests are still very different from all other mentioned forms of information update: besides their epistemic effects, they always have an ontic impact as well. In a quantum world, there is no "purely epistemc" action, no free informational lunches, no pure observations: all epistemic actions have ontic side-effects. Sixth, these ontic side-effects of quantum tests have non-classical epistemic consequences, leading to a non-monotonic dynamics of information. Another paradigmatic case of non-monotonic information change is belief revision: as in quantum tests, prior information can be overwritten by new incoming information. (But note that in the first case the actual ontic state changes due to quantum tests while in the latter case only the doxastic state is changed.) Finally, the ontic side-effects of quantum tests are 
non-local: observing a nearby (sub)system may change (sub)systems that are far away! In the quantum world, learning is not a local affair: more precisely, although all learning is local in its content (since only local properties can be learnt, due to the relativistic limit to information flow), the act of learning such local features can have global impact.

\section{Conclusion}

We have taken "quantum dynamic logic" to be the logic of physically-constrained informational interactions. These informational interactions took the form of measurements as well as entanglement between the subsystems of a given global physical system. Similarly, (multi-agent) epistemic logic has been approached as the logic of socially-constrained informational interactions (in the form of direct observations, learning, various forms of communication and testimony, and more complex forms of group knowledge-aggregation) between "subsystems" of a social system. Analyzing both areas from the point of view of informational interactions has provided us with a unifying perspective, allowing us to compare various forms of information update.

This view on the dynamic nature of informational interactions has opened up several roads for further research in different directions: 1) it has provided a multiagent perspective to belief revision theory and given new tools to model relevant phenomena in AI and social epistemology; 2) it has provided new formal tools that can be used to give a dynamic account of knowledge in formal epistemology, one direction that is currently being further explored defines knowledge as a fixed-point of epistemic actions [3]; 3) the epistemic multi-agent perspective and the quantum dynamic framework are logically compatible and can be united to provide an integrated account to model both classical and quantum interactive scenarios.

There are limits to the power of these formalisms, but these limits provide challenges and opportunities for further logical investigation. In particular, most quantum communication protocols known today are multi-agent applications that involve both a quantum information flow and a classical knowledge transfer (by classical communication) between agents. However the formal tools used to model these applications within quantum information theory, cannot fully account for such intricate classicalquantum interactions. A more sophisticated combination of Dynamic Quantum Logic and DEL is thus needed for the full specification and formal verification of agentbased quantum protocols (such as Teleportation). In recent and on-going work [19], we use a probabilistic extension of Quantum Dynamic Epistemic Logic, employing quantum event models, to capture this complex interplay between classical and quantum informational flow.

Finally, we would like to stress that, while quantum tests do share some formal features with purely epistemic/doxastic actions in Belief Revision theory and public announcement operators, we are not trying to explain quantum behavior as a purely epistemic or informational artifact (as some other authors have attempted, e.g. [38]). In our view, quantum weirdness is not just a product of the observer's mind. On the contrary, we adopt a realist point of view: it is precisely the realworld changes induced by quantum tests that are responsible for their epistemic 
peculiarities. Quantum learning is characterized by the erosion of the sharp classical separation between "ontic" and "epistemic" : as we saw, in the quantum world there can be no information change without changing the world [10]. Moreover, the realworld effects of such information-gathering actions cannot be confined to a localized region. As we saw, this is the "essence" of entanglement viewed from in an abstract dynamic-logical form. Modern quantum computation theory takes this as an opportunity, rather than a problem or a weird case of spooky witchcraft: entanglement is a resource which, if appropriately used, holds the promise of giving us new, almost magical (though not unlimited) informational capabilities. And quantum dynamic epistemic logic gives us both a better understanding of the nature of this resource and a warning about its dangers. In the end, it shows that any attempts to learn more about our immediate environment have unintended and far-reaching effects, going far beyond our local circle. Entanglement and non-locality can thus be understood as just more radical (and more realistic) physical embodiments of the above-mentioned principle that "epistemic actions have ontic side-effects". The whole universe is being changed by our seemingly provincial squabble for information.

Acknowledgments The contribution of Sonja Smets was funded in part by the VIDI grant 639.072.904 of the Netherlands Organisation for Scientific Research and by the European Research Council under the 7th Framework Programme FP7/2007-2013/ERC Grant no. 283963.

Open Access This article is distributed under the terms of the Creative Commons Attribution License which permits any use, distribution, and reproduction in any medium, provided the original author(s) and the source are credited.

\section{References}

1. Alchourron, C.E., Gärdenfors, P., \& Makinson, D. (1985). On the logic of theory change: partial meet contraction and revision functions. Journal of Symbolic Logic, 50, 510-530.

2. Baltag, A. (2002). A Logic for Suspicious Players: Epistemic Actions and Belief Updates in Games. Bulletin of Economic Research, 54(1), 1-45.

3. Baltag, A., van Benthem, J., \& Smets, S. Music of knowledge. book manuscript in preparation.

4. Baltag, A., \& Moss, L. (2004). Logics for Epistemic Programs. Synthese, 139, 165-224.

5. Baltag, A., Moss, L.S., \& Solecki, S. (1998). The logic of public announcements, common knowledge, and private suspicions. In I. Gilboa (Ed.) Proceedings of TARK (Vol. 98, pp. 43-56).

6. Baltag, A., Moss, L., \& van Ditmarsch, H. Epistemic logic and information update. Handbook on the Philosophy of Information.

7. Baltag, A., \& Smets, S. (2005). Complete axiomatizations of quantum actions. International Journal of Theoretical Physics, 44(12), 2267-2282.

8. Baltag, A., \& Smets, S. The logic of quantum programs. In P. Selinger (Ed.) Proceedings of the 2nd International Workshop on Quantum Programming Languages (QPL2004), TUCS General Publication (Vol. 33, pp. 39-56). Turku Center for Computer Science, 2004. PHILSCI00001799.

9. Baltag, A., \& Smets, S. (2006). LQP: The dynamic logic of quantum information. Mathematical Structures in Computer Science, Special Issue on Quantum Programming Languages, 16(3), 491-525.

10. Baltag, A., \& Smets, S. (2008). A dynamic-logical perspective on quantum behavior. Studia Logica, special issue on Applied Logic in the Philosophy of Science, 89, 185-209.

11. Baltag, A., \& Smets, S. (2011). Quantum logic as a dynamic logic. Synthese, 179(2), 285-306.

12. Baltag, A., \& Smets, S. (2010). Correlated knowledge, an epistemic-logic view on quantum entanglement. International Journal of Theoretical Physics, 49(12), 3005-3021.

13. Baltag, A., Bergfeld, J., Kishida, K., Sack, J., Smets, S., \& Zhong, S. (2014). PLQP \& company: decidable logics for quantum algorithms. International Journal of Theoretical Physics, 53(10), 36283647 . 
14. Baltag, A., Bergfeld, J., Kishida, K., Sack, J., Smets, S., \& Zhong, S. (2013). Quantum probabilistic dyadic second-order logic. In L. Libkin, U. Kolhenbach, \& R. de Quieroz (Eds.) Wollic, 2013 Volume, LNCS (Vol. 8071, pp. 64-80).

15. Baltag, A., \& Smets, S. (2006). Conditional doxastic models: a qualitative approach to dynamic belief revision. In G. Mints, \& R. de Queiroz (Eds.) Electronic notes in theoretical computer science (Vol. 165, pp. 5-21).

16. Baltag, A., \& Smets, S. (2006). The logic of conditional doxastic actions: a theory of dynamic multiagent belief revision. In S. Artemov, \& R. Parikh (Eds.) Proceedings of the Workshop on Rationality and Knowledge, ESSLLI.

17. Baltag, A., \& Smets, S. (2006). Dynamic belief revision over multi-agent plausibility models. In G. Bonanno, W. van de Hoek, \& M. Woolridge (Eds.) 7th Conference on logic and the foundations of game and decision. Liverpool.

18. Baltag, A., \& Smets, S. (2008). A qualitative theory of dynamic interactive belief revision. In G. Bonanno, W. van der Hoek \& M. Wooldridge (Eds.), Logic and the foundations of game and decision theory, texts in logic and games (vol. 3, pp. 958). Amsterdam University Press.

19. Baltag, A., \& Smets, S. (2014). Probabilistic dynamic epistemic logic: classical and quantum, presentation given at the Amsterdam Quantum Logic Workshop. The Netherlands.

20. van Benthem, J. (2006). Dynamic logic for belief revision, ILLC Tech Report. DARE electronic archive: University of Amsterdam. Printed in Journal of applied non-classical logics.

21. van Benthem, J. (2007). Dynamic logic for belief revision. Journal of Applied Non-classical Logics, 17(2), 129-155.

22. van Benthem, J. (2011). Logical dynamics of information and interaction: Cambridge University Press.

23. van Benthem, J., van Eijck, J., \& Kooi, B. (2006). Logics of communication and change. Information and Computation, 204(11), 1620-1662.

24. Birkhoff, G., \& von Neumann, J. (1936). The logic of quantum mechanics. Annals of Mathematics, 37, 823-843.

25. van Ditmarsch, H. Knowledge games. PhD thesis, University of Groningen, 2000. ILLC Dissertation Series DS-2000-06.

26. Fagin, R., Halpern, J., Moses, Y., \& Vardi, M. (1995). Reasoning about knowldege: MIT Press.

27. Foulis, D.J., \& Randall, C.H. (1974). Empirical logic and quantum mechanics. Synthese, $29,81$.

28. Gerbrandy, J.D. Bisimulations on Planet Kripke. PhD thesis, University of Amsterdam, 1998. ILLC Dissertation Series DS-1999-01.

29. Gerbrandy, J.D., \& Groeneveld, W. (1997). Reasoning about information change. Journal of Logic, Language, and Information, 6, 147-169.

30. Harel, D., Kozen, D., \& Tiuryn, J. (2000). Dynamic logic: MIT Press.

31. Jauch, J.M. (1968). Foundations of quantum mechanics. Reading: Addison-Wesley.

32. Jauch, J.M., \& Piron, C. (1969). On the structure of quantal proposition systems. Helvetica Physica Acta, 42, 842-848.

33. Piron, C. (1964). Axiomatique quantique (PhD-Thesis). Helvetica Physica Acta, 37, 439-468. English Translation by M. Cole: "Quantum Axiomatics" RB4 Technical memo 107/106/104, GPO Engineering Department London.

34. Piron, C. (1976). Foundations of quantum physics. Massachusetts: W. A. Benjamin.

35. Plaza, J.A. (1989). Logics of public communications. In M.L. Emrich, M.S. Pfeifer, M. Hadzikadic $\&$ Z.W. Ras (Eds.), Proceedings of the 4th International symposium on methodologies for intelligent systems, (pp. 201216).

36. Putnam, H. (1968). Is logic empirical? In R. Cohen, \& M. Warofsky (Eds.) Boston studies in the philosophy of science Vol. 5. Dordrecht: Reidel.

37. Randall, C.H., \& Foulis, D.J. (1979). The operational approach to quantum mechanics. In Hooker, C.A. (Ed.) Physical theory as logico-operational structure. Dordrecht: D. Reidel Publishing Company.

38. Spekkens, R.W. (2005). In defense of the epistemic view of quantum states: a toy theory. arXiv:quan$\mathrm{t}-\mathrm{ph} / 0401052 \mathrm{v} 2$.

39. von Neumann, J. (1932). Mathematische Grundlagen der Quantenmechanik. Berlin: Springer. (English translation: Mathematical Foundations of Quantum Mechanics, Princeton University Press, Princeton, 1955). 